\title{
Reconfigurable fixture evaluation for use in automotive light assembly
}

\author{
Martin Bem, Miha Deniša, Timotej Gašpar, Jaka Jereb, Robert Bevec, Igor Kovač and Aleš Ude \\ Department of automatics, biocybernetics and robotics \\ "Jožef Stefan" Institute \\ Jamova cesta 39, 1000 Ljubljana, Slovenia \\ \{martin.bem, miha.denisa, timotej.gaspar, jaka.jereb, robert.bevec, igor.kovac ales.ude\}@ijs.si
}

\begin{abstract}
To make robotics feasible for use in small and medium enterprises (SMEs) several issues have to be addressed. The most obvious is the ability to produce small batches of products with minimal changeover cost and time. In this paper an innovative flexible fixture based on a Gough-Stewart platform called the hexapod is proposed. The fixture is designed to be suitable for a whole family of automotive lights. The main characteristics of the hexapod is its passivity, i.e. the reconfiguration can be performed manually or by using an external mechanism e.g. a robot. Once a desirable configuration is reached a set of hydromechanical brakes is used to hold the mechanism in the desired pose. To show the effectiveness of the hexapods a set of automotive lights assembly in dedicated robot cell experiments were performed. Additionally, positioning repeatability and the locked system stiffness were measured. Finally, a robot cell for a complete light assembly has been implemented together with robot assisted fixture reconfiguration between different light models.
\end{abstract}

Index Terms-Reconfigurable fixture, robotic assembly, automotive lights

\section{INTRODUCTION}

One of the vital aspects in manufacturing is fixing a workpiece in place during the machining or assembly processes. This is usually done by fixtures, which are essential elements in most production processes. Their design is vital, as they influence the productivity, the cost, and the product quality. It is estimated that $40 \%$ of rejected parts stem from the inappropriate fixturing [1].

Fixturing systems can be divided into two major categories: dedicated and flexible systems [2]. The majority of currently used fixtures are dedicated, as they are designed for a specific part and/or operation [3]. A single-purpose approach of dedicated fixtures introduces long lead time and high efforts required for design and manufacturing. In addition, each modification of parts and/or operations leads to a manual set-up and the need to store and retrieve dedicated fixtures. All this increases the cost related to dedicated fixtures. Typically, the costs associated with dedicated fixtures can represent up to $10-20 \%$ of the total costs of a manufacturing system [4]. Long lead times can also

This work has been funded by the Horizon 2020 ICT-FoF Innovation Action no. 680431, ReconCell (A Reconfigurable robot workCell for fast set-up of automated assembly processes in SMEs).

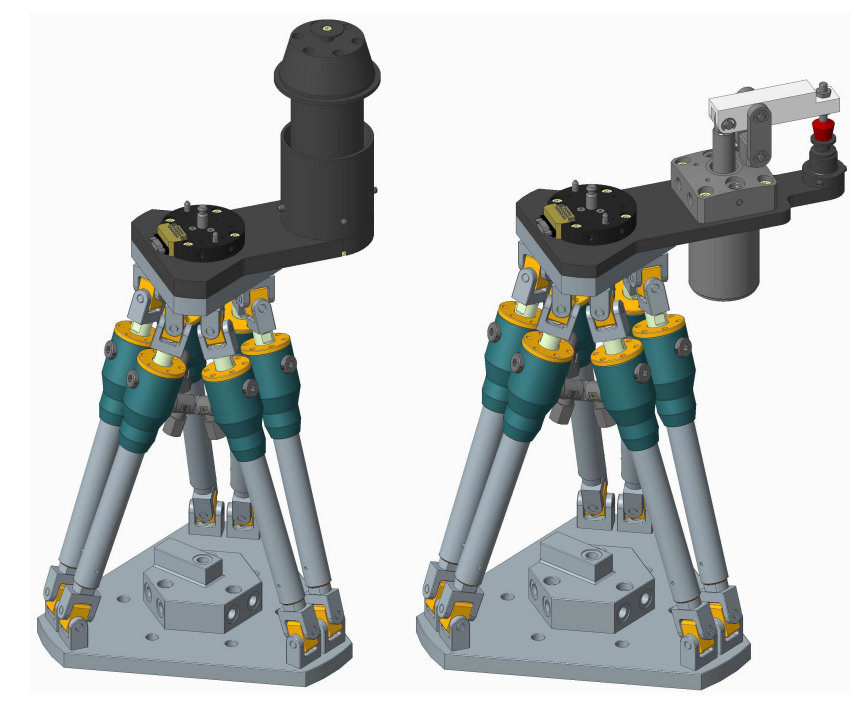

Fig. 1. Flexible fixture consisting of a hexapod, tool changer connector, and two examples of modular workpiece locating assembly.

cause a loss of opportunity in volatile markets. This means that a dedicated fixturing system is feasible only for mass production, where the production volumes are high while the product variety is low.

On the other hand, flexible fixtures, are designed to be reusable and more general purpose. A truly general purpose fixture is unfeasible, due to an almost infinite variety of workpiece geometries and operations requirements. Therefore, flexible fixtures are designed to fit a family of resembling products which need similar manufacturing operations. Such a reconfigurable approach reduces the design complexity and costs, compared to the fully flexible approach, while still providing enough flexibility. Compared to the dedicated fixtures, the initial costs of flexible fixtures are higher, but the long term overall production costs are lowered significantly due to fixture reuse [1]. Flexible fixtures can be a part of reconfigurable systems, which are designed to provide desired capacity and functionality whenever needed [5]. This reconfiguration can be achieved with or without worker assistance. Reconfiguration approach is a key enabling technology 
for the introduction of automation into small and medium enterprises (SMEs) where product variety is high and batches are small.

We propose to use a robotically reconfigurable fixture based on an unactuated Gough-Stewart parallel mechanism. The positioning of the fixture (workpiece) is done by a robot. The case study tackled in this paper encompasses an assembly of a family of automotive light casings. The fixture is designed and tested on it. In addition, measurements of position repeatability and resistance to external forces were performed.

The further structure of the paper is as follows. While section 2 looks at related works, Section 3 presents an overview of the proposed approach. Evaluation, which is done in two parts, is presented in Section 4. Concluding remarks are given towards the end of the paper.

\section{RELATED WORK}

Different solutions for reconfigurable and flexible fixturing solutions are described in literature. Asada and By [6] describe a robotically reconfigurable modular fixture system. The system uses different clamping modules (e.g. vertical clamps, horizontal clamps) attachable with magnetic chucks to a table. Depending on the workpiece geometry the robot can reposition the modules and then lock them in place. The system was developed for a small batch production where batch sizes range between 20 and 100. The use of magnetic chucks makes the system useful only for nonmagnetic workpieces. A modular programmable conformable clamping system for fixturing a variety of turbine blades during machining was developed by Kurokawa [7]. It uses a hinged octagonal frame positioned around an arbitrary section alongside the turbine blade. The lower half of the frame employs pneumatic plungers which in released state are free to conform to the turbine blade profile. Then, a high strength belt is used to press the convex part of the blade against the locked plungers. The most flexible approach is to use special robots as fixtures. Specially designed servo actuators were proposed for work-holding by [8] and [9]. These solutions are the only ones enabling production down to the lot size of one. However, due to the high initial costs, such solutions are unfeasible for SMEs. A cheaper passive modular system for flexible assembly of square tubes was presented in [10]. This system replaces traditional fixtures that are product specific, permanent, time consuming and costly with a new modular and flexible system that can be rapidly and inexpensively redesigned to fit to the new product. If production is relaunched fixtures must be stored and this requires a lot of storage area. If the reconfigurable fixtures are used, this is not a problem since one fixture serves for many products and in the case of production relaunch, the fixture is simply reconfigured. Flexible fixtures developed by [11] are based on GoughStewart parallel mechanism i.e. hexapods. They can be moved in all six spatial degrees of freedom. The used hexapods are unactuated and must be manually moved by hand or robot and then locked in desired position with a locking sleeve or with help of hydraulic system. The hexapods offer high flexibility and rapid changeovers.

\section{FleXible FIXTURE}

Our fixture, i.e. the hexapod, is based on an unactuated Gough-Stewart parallel mechanism (see figure 1). The hexapod uses two plates connected by six links. The base plate is rigidly connected to the robotic cell. The top plate is equipped with a modular workpiece locating assembly and with a tool side of the robotic tool changer connector. The connector enables that the reconfiguration can be performed by an external mechanism, e.g. a robot. Each of the six links of the parallel mechanism is composed of two special preloaded Cardan joints and a prismatic joint with an integrated hydromechanical brake. The Cardan joints connect the link to the top and base plate and are specially designed to minimize backlash. The prismatic brake is used to lock the mechanism in place once a suitable configuration has been achieved. Such a configuration gives the top plate the ability to move in all six spatial degrees-of-freedom. The mechanism is the final result of on extensive research done previously [12], [13], [14]. The hexapod used in this research was redesigned based on the required workspace for fixturing the automotive lights family.

The workpiece locating assembly is the part of the fixture that is in a direct physical contact with the workpiece. Its design depends heavily on workpiece geometry and manufacturing operations. Therefore a flexible, entirely universal solution cannot be found. Instead, the reconfiguration approach offering a limited flexibility regarding the workpiece family is more feasible.

For use in automotive light assembly, workpiece locating was designed based on the analysis of the common geometric features of the whole product family. It was determined that mounting holes were a suitable feature for locating and clamping the workpiece. However, different models have different hole diameters. To make the locating assembly compliant with the whole parts family, the centering pins are interchangeable. In order to minimize the human interaction and speed up the reconfiguration process the pins are exchangeable by a robot. A pneumatic lever clamp is used for securing the workpiece in place.

Preliminary testing revealed that additional support was needed during assembly operation. A new locating assembly that used a centering element was designed to be used as a supplement to the clamping system. The centering element locates and supports the hole for the main bulb of the automotive light. Different models have different diameter holes so the seating elements are exchangeable by robot as well. The experimental implementation for automotive assembly is described in detail in Subsection IV-B. 

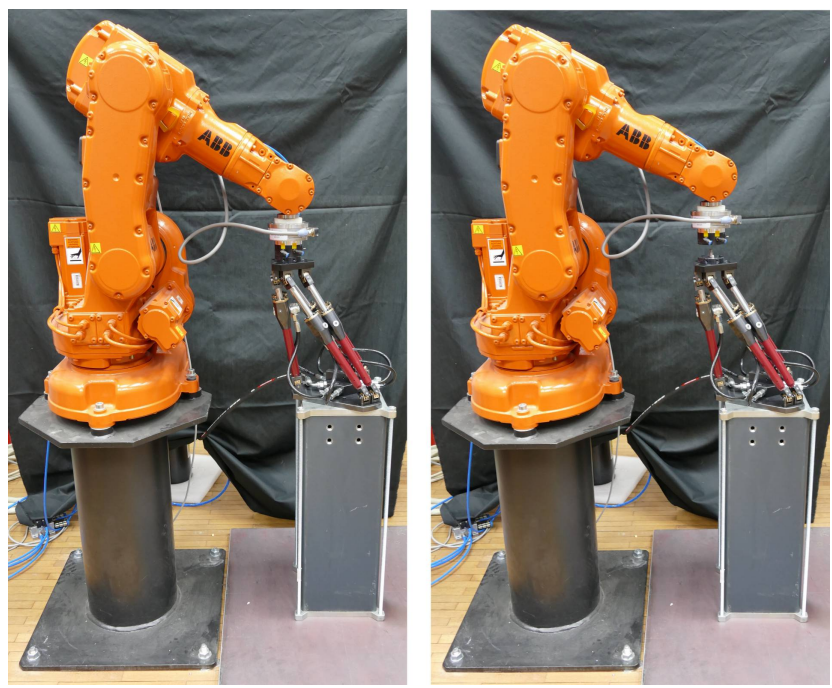

Fig. 2. Hexapod evaluation setup. First the robot moves the compliant hexapod to a new position (left image). The hexapod top plate position is measured. The hexapod brakes are activated and the robot releases it (right image). The top plate position is measured again.

\section{EVALUATION}

Three different aspects of flexible fixtures were evaluated. Firstly, hexapod mechanism was evaluated. We evaluated the stiffness of the locked mechanism and position accuracy while repositioning it. The proposed flexible fixtures we evaluated in a case study based on a real industry scenario.

\section{A. Hexapod Evaluation}

To use in a robot assembly application, the reconfigurable fixture has to satisfy performance requirements. The most important is the positioning precision that can be achieved by the parallel mechanism. Generally, the workpiece can be supported by multiple reconfigurable fixtures thus the absolute positioning accuracy becomes relevant. Since an external mechanism, e.g. robot, is used to perform the reconfiguration the absolute fixture configuration accuracy is the same as the accuracy of the robot. As the fixture is intended to be used in robotic cells where the reconfiguration is performed by the same robot as for performing the assembly process, we can assume that a robot with adequate accuracy to perform the assembly processes will be sufficiently accurate to configure the fixtures. Therefore, the absolute accuracy of the hexapod mechanism is not the focus of this paper.

However, the position locking repeatability is directly determined by the hexapod locking mechanism. When the hexapod brakes are released and the hexapod is compliant it is positioned to a desired configuration by the robot. When the brakes activate there is a force acting upon the rods in the hexapods links. If locking the brakes disrupts the final position of the fixture, it cannot be properly positioned regardless of robot positioning accuracy. Consequently, the

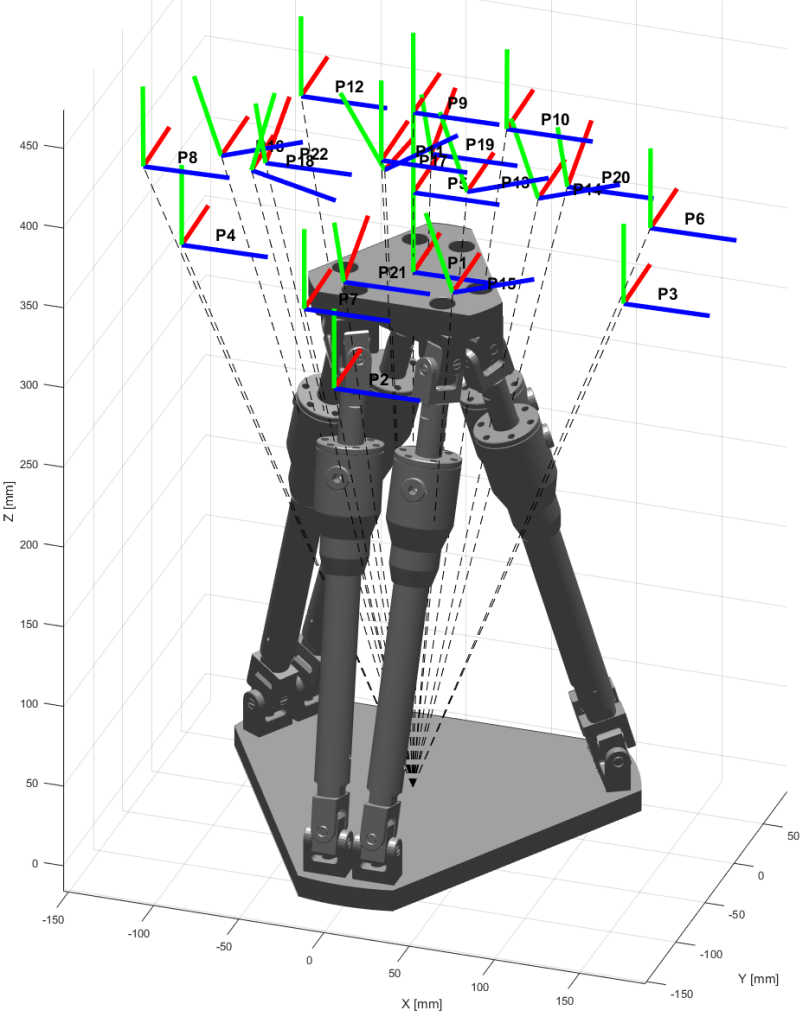

Fig. 3. Locations of top plate used in the measurement of hexapod repeatability

workpiece will not fit or will be misaligned and any further assembly operations will be impossible.

Another important characteristic is the stiffness of the locked hexapod mechanism. During the robotic assembly operations forces are exerted on the workpiece that are consequently transmitted to the fixtures. If the fixture is not stiff enough the exerted forces displace the fixture and the workpiece on it.

To determine the above described parameters the proposed fixture was experimentally tested. The main goal was to determine whether the reconfigurable fixture can be reliably used in robotic assembly cells. The test setup, seen in Fig. 2 , was composed of a fixture with the baseplate attached to a stiff metal pillar attached to the floor. An ABB IRB 140 with a ATI Delta force torque sensor and a Destaco QC-30 tool changer robot adapter module attached to the tip was used. The TP-30 tool changer tool adapter module was mounted to the hexapods top plate. To facilitate the position measuring no locating assembly was used. To measure the position of specified points on the hexapod a GOM Athos CS 5m optical measuring system was used with a measurement uncertainty 


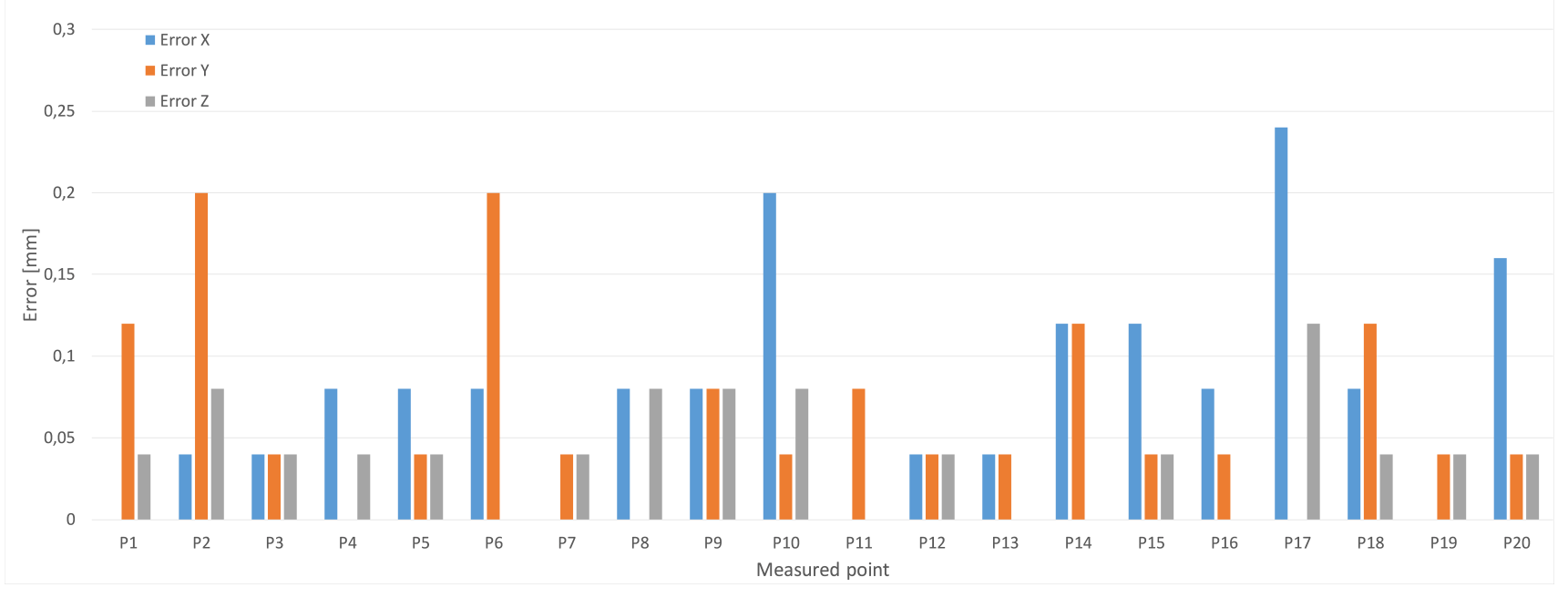

Fig. 4. Position errors after locking the mechanism for all 20 measured poses (Note that errors lower than measuring accuracy were set to zero).

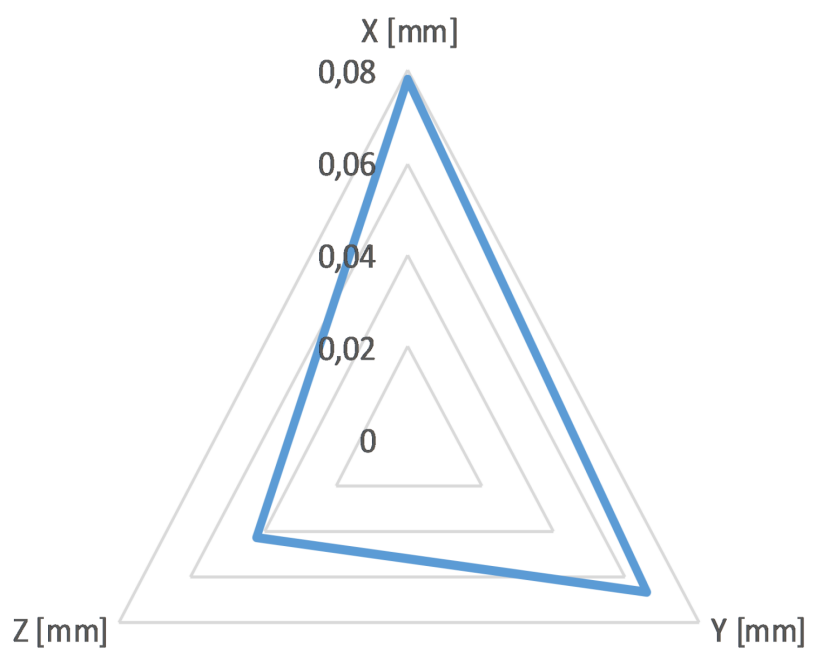

Fig. 5. Average position repeatability in spatial directions after locking

of $0.04 \mathrm{~mm}$. It uses a stereo triangulation to obtain the surface data in a form of a point cloud. For each measurement 3-5 scans were made to get enough geometric data of the points on the top and bottom hexapod plate. From the measured data three points on the hexapods top plate were extracted and their positions relative to the bottom plate coordinate system have been calculated. By measuring three different points on the hexapods platform we were able to determine the positional and rotational displacements relative to the hexapod base.

1) Position Repeatability: The positioning repeatability was measured in 20 different configurations as shown on Fig. 3. The selected points were distributed equally inside the workspace envelope so that we could measure the effect of the brakes actuation throughout the entire hexapod workspace. The robot was used to reconfigure the hexapod by connecting the robot adapter tool changer to the tool adapter module on the hexapod's top plate. The reference position and orientation was measured twice first while the hexapod was connected to the robot and was still compliant. Afterwards, the mechanism was locked, the tool changer uncoupled and the tip of the robot retracted. Then the second position measurement was made to obtain the position and orientation of the top plate. In this way we were able to determine if the hexapod locking has any influence on the positional accuracy.

To determine the positional and orientation repeatability we did statistical analysis of the measured data (20 measurement points). The obtained result are presented in Figs. 4 and 5. The mean positional displacement due to the position locking was $0.08 \mathrm{~mm}$ with standard deviation of $0.08 \mathrm{~mm}$. The mean value of the rotational displacement was $0.9 \mathrm{mrad}$ and with standard deviation of $0.0005 \mathrm{mrad}$.

During the evaluation negative effects of using the tool changer to connect to the hexapod were observed. During the uncoupling process the plates are pushed apart for approximately $1 \mathrm{~mm}$. The force is considerable and caused visible deformations of the robot. The problem could be solved by using an electromagnetic gripper.

The biggest displacements were observed in the $\mathrm{x}$-direction and the smallest in the z-direction regarding the hexapod base coordinate system (figure 5). This is a consequence of nonlinearity of the mechanism stiffness in different directions and configurations [15]. Because the force resulting from opening the tool acts perpendicularly to the top plate forces in different directions are exerted on the hexapod mechanism links. When a load is applied in the $\mathrm{z}$-direction the the angles 


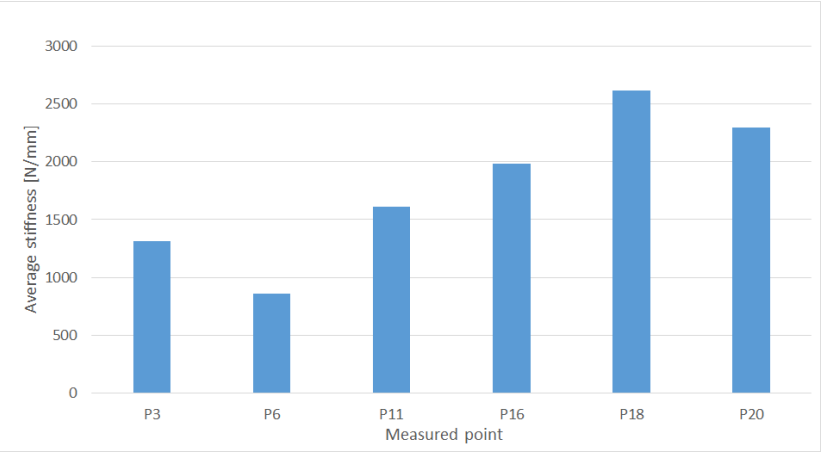

Fig. 6. Average stiffness in measured points

between the mechanism links and the force acting on them are generally smaller than in the $\mathrm{x}$ and $\mathrm{y}$ directions resulting in better stiffness and locking repeatability.

The measuring accuracy of the used GOM system is to low to reliably measure the repeatability of the fixture. The fixture's performance exceeded our expectation. Therefore, more precise measurements are needed to determine the numerical values of the locking repeatability. The presented values were only for evaluating the systems usability in our use case. The considered assembly task are included and the light housing mounting holes are produced to a tolerance similar to the measured unrepeatability. Hence, the fixture can be reliably used in the use case.

2) Stiffness: The stiffness was measured in 6 of the 20 points used for the repeatability measurement. The hexapod was locked and forces were applied in 6 directions (positive and negative translation along all axes of the hexapod base coordinate system and positive and negative rotation along the $\mathrm{Z}$ axis) using the robot connected to the hexapod with the tool changer. Forces were generated by pushing the robot 0.3 $\mathrm{mm}$ in each direction while the mechanism was locked. The force magnitudes varied deepening on the combined stiffness of the robot and the hexapod in a given configuration and direction. Forces and torques were measured using a force torque sensor mounted to the robot wrist. A set of GOM measurements was made before and after the application of the force in each direction to determine the magnitude and direction of displacements. The position and orientation of the top plate was measured relative to the hexapod bottom plate to avoid measuring the deformation of other components, e.g. the pillar.

These measurements give us an overview of the stiffness characteristics of the hexapod throughout its workspace. The siffness was defined as

$$
k=\frac{F}{\delta}
$$

where $F$ the force measured using the force/torque sensor and $\delta$ the deformation measured using the GOM system.

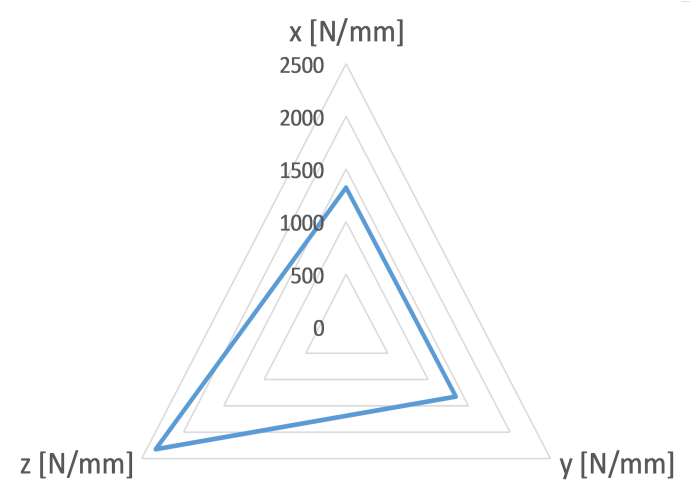

Fig. 7. Average stiffness in spatial directions

Individual stiffnesses were calculated taking into account only the components of forces and deformations collinear to the direction of external load. The average stiffness values in each point are given in Fig. 6. The average stiffness across all the points in all directions was approx. $1780 \mathrm{~N} / \mathrm{mm}$.

Different average stiffnesses were observed in different directions. Average stiffness in the $\mathrm{x}$ and $\mathrm{z}$ direction were measured to be $1328.8 \mathrm{~N} / \mathrm{mm}$ and $1333.4 \mathrm{~N} / \mathrm{mm}$, respectively. The values are similar because of the symmetry of the mechanism. In the $\mathrm{z}$ direction the stiffness was considerably higher and was measured to be $2323.4 \mathrm{~N} / \mathrm{mm}$. This is to be expected as the angle between the hexapod legs and load in this direction is the smallest. A bigger stiffness in $\mathrm{z}$ direction is beneficial for our case because most assembly operations exert forces along this axis.

\section{B. Case Study Evaluation}

For the evaluation of the fixture we have selected case examples from the automotive light industry where a variety of different automotive lights are manufactured. The assembly process starts by injection molding the light housing. After a cool-down period the housing is manually inserted in an assembly device that mounts assembly components such as light height adjustment motor, bulb holder and a sheet metal heat shield, depending on the housing model. In the assembly process a self tapping screw is also screwed using a servo screwdriver. Currently each model requires two assembly devices (mirrored), one for the left and one for the right light housing. When a new model is introduced, a new assembly device must be designed and manufactured. This increases the production lead times and raises costs. However, when an old part goes out of the production the associated assembly machine can not be dismantled immediately. The assembly machines must be kept in storage for about 10-15 years for the production of spare parts in small batches (a couple of times per year). Since the company produces multiple models of light housing at the same time, storing different assembly machines becomes expensive. 

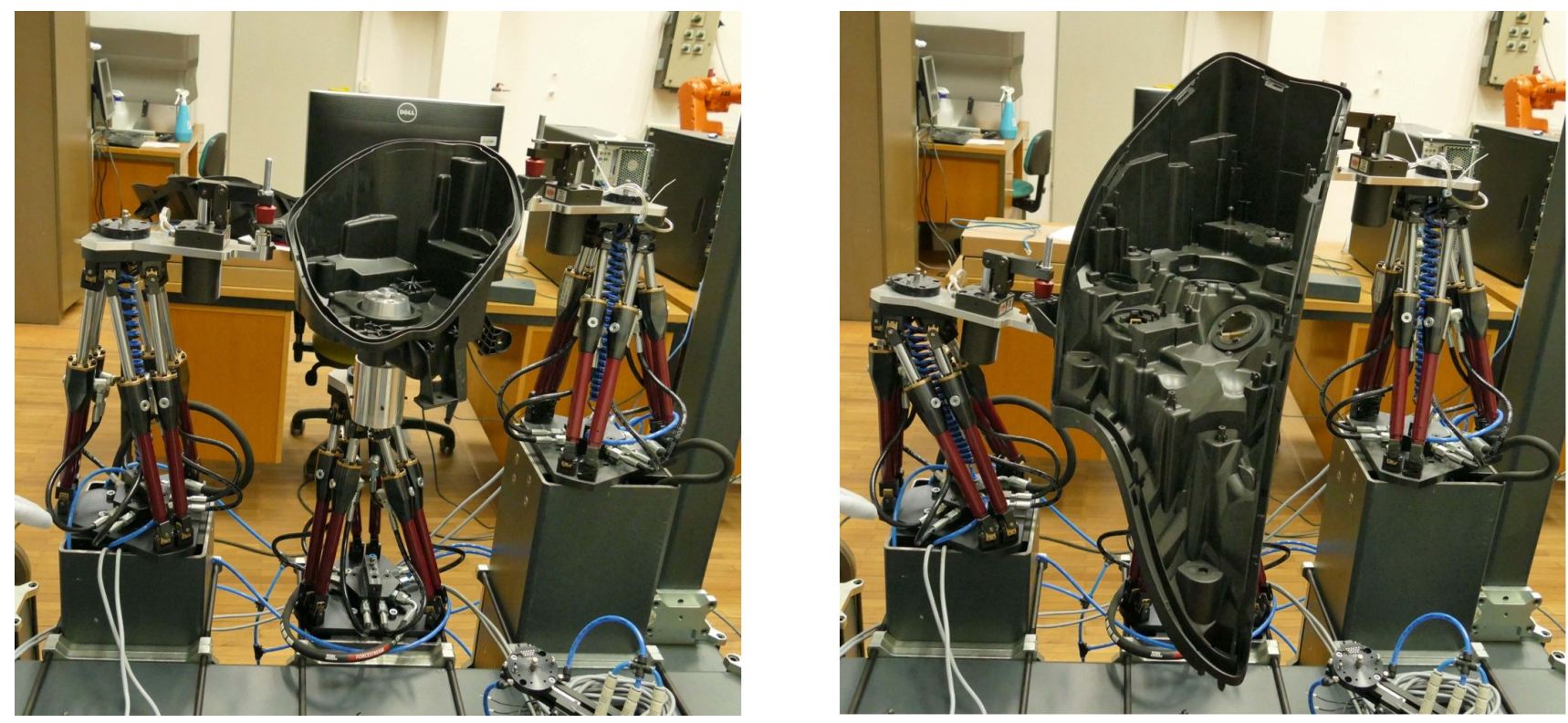

Fig. 8. Reconfigurable fixtures holding x07 light housing (left) and x82 light housing (right)

The main goal of this case study is to implemented and test flexible robotic assembly cells equipped with flexible passive fixtures, which are capable of assembling the whole family of automotive lights with minimal changeover time and cost. Two different automotive lights (shown in Fig. 8) were chosen for the first experiments. They were selected as they have very different geometry and still require similar assembly operations. In this way we can demonstrate the flexibility and reconfigurability of the robotic cell.

A total of three reconfigurable fixtures are used for workholding. Two of them are equipped with pneumatic clamping and one with centering element locating assembly. Changeover between the models requires in the first step repositioning of the thee hexapods to a predetermined model specific configuration. Afterwards the centering pins of the clamping assembly and the centering elements are removed and stored in a specially designed pallets using a dedicated robotic end effector. Finally, new centering elements are installed.

The robotic reconfiguration was successfully tested multiple times. An Universal Robots UR10 robot was used to move the hexapods. The exchange of modular elements was performed manually. The fixture reconfiguration results are shown in Fig. 8.

Preliminary experiments of robotic assembly using the proposed fixtures were also performed. The $\mathrm{x} 07$ workpiece was chosen for assembly testing. The assembly operations consisted of performing the whole assembly task (currently performed by the model specific assembly machine) with the workpiece held in place by the reconfigurable fixtures. Several assembly cycles were successfully performed. The insertion of the light housing was reliable even when gripping was suboptimal. No accuracy issues were observed while installing assembly components. The forces exerted on the workpiece did not result in any observable fixture deformation. Although testing is in a preliminary stage and further experiments are required, the reconfigurable fixtures show great potential to reduce the fixture costs and to shorten the changeover times.

\section{CONClusion}

Hexapods were evaluated in terms of repeatability and stiffnesses. We showed the average locking repeatability in multiple axes was $0.08 \mathrm{~mm}$. While a more precise measurements would be beneficial, current measurements support the viability of using the proposed hexapods for automotive light assembly. The stiffness of a locked hexapod was also measured. While the stiffness in two of the axis was 1328.8 $\mathrm{N} / \mathrm{mm}$ and $1333.4 \mathrm{~N} / \mathrm{mm}$, the stiffness in axis, were the most assembly operations apply forces, was even greater (2323.4 $\mathrm{N} / \mathrm{mm}$ ).

A case study, based on a current assembly process in an SME, was used to evaluate the proposed flexible fixtures. We showed the fixtures are adaptable enough to firmly mount two fairly different automotive light casing. Multiple executions of the assembly procedures showed the reliability of the fixtures. Even when force are applied due to interaction between the robot and the item that has to be assembled, the displacement is small enough that it does not affect the assembly process.

To further show the benefits of using reconfigurable fixtures additional experiments are needed. Using a tool changer 
proved to be non optimal and may be partially responsible for the observed locking inaccuracies. Measuring the repeatability of the brakes using an electromagnetic chuck to move the hexapod would minimize external forces and enable us to better determine the actual locking repeatability. Repeating the experiments using a measuring method with better spatial resolution would also be beneficial. Measuring a stress-deformation characteristic in each axis would also be interesting as it would help us determine the source of displacements. The assembly of the x 82 light housing and the automatic reconfiguration between models will be implemented and tested.

\section{REFERENCES}

[1] H. Wang, Y. K. Rong, H. Li, and P. Shaun, "Computer aided fixture design: Recent research and trends," Computer-Aided Design, vol. 42, no. 12, pp. 1085-1094, 2010.

[2] Z. Li, Z. J. Pasek, and J. Adams, "Reconfigurable fixtures: Concept and examples," in In Proc. JUSFA, (Denver, Colorado, USA), 2004.

[3] A. Y. C. Nee, Z. J. Tao, et al., An advanced treatise on fixture design and planning, vol. 1. World Scientific, 2004.

[4] Z. Bi and W. Zhang, "Flexible fixture design and automation: review, issues and future directions," International Journal of Production Research, vol. 39, no. 13, pp. 2867-2894, 2001.

[5] Y. Koren, U. Heisel, F. Jovane, T. Moriwaki, G. Pritschow, G. Ulsoy, and H. Van Brussel, "Reconfigurable manufacturing systems," CIRP Annals-Manufacturing Technology, vol. 48, no. 2, pp. 527-540, 1999.

[6] H. Asada and A. By, "Kinematic analysis of workpart fixturing for flexible assembly with automatically reconfigurable fixtures," IEEE Journal on Robotics and Automation, vol. 1, no. 2, pp. 86-94, 1985.
[7] E. Kurokawa, "Flexible conformable clamps for a machining cell with applications to turbine blade machining.," tech. rep., DTIC Document, 1983.

[8] I. Lütkebohle, "GM Launches Flexible Tooling for Robotic Welding Automation World." https://www. automationworld.com/lean-manufacturing/ gm-launches-flexible-tooling-robotic-welding-0/, 2008. [Online; accessed 2017-04-11].

[9] G. S. Vasilash, "GM + NASA = Remarkable Robots: Automotive Design \& Production." http: / /www . adandp.media/blog/post/ gm-nasa-remarkable-robots, 2010. [Online; accessed 201704-11].

[10] I. Erdem, P. Helgosson, and H. Kihlman, "Development of automated flexible tooling as enabler in wing box assembly," Procedia CIRP, vol. 44, pp. 233-238, 2016.

[11] H. Kihlman, Affordable automation for airframe assembly: developing of key enabling technologies. $\mathrm{PhD}$ thesis, Linköping Univinversity, Linköping, Sweden, 2005.

[12] K. Salminen and I. Kovač, "Role based self-adaptation of reconfigurable robotized systems for sustainable manufacturing," 22nd International Conference on Flexible Automation and Intelligent Manufacturing, FAIM, jun 2012.

[13] W. Fritz and I. Kovač, "An approach for shim-less tooling using robot guided reconfiguration system," in In Proc. JUSFA, (Denver, Colorado, USA), 2004.

[14] M. Gödl and I. Kovač, "A robot guided reconfigurable assembly system," in In Proc. CIRP, (Ann Arbor, Michigan, USA, Ann Arbor), 2005.

[15] A. Pashkevich, A. Klimchik, and D. Chablat, "Nonlinear effects in stiffness modeling of robotic manipulators," in International Conference on Computer and Automation Technology, (Venice, Italy), pp. 168-173, 2009. 